\title{
Structural diversity of disc galaxies originating in the cold gas inflow from cosmic webs
}

\author{
Masafumi Noguchi ${ }^{1 \star}$ \\ ${ }^{1}$ Astronomical Institute, Tohoku University, 6-3, Aramaki, Aoba-ku, Sendai, Miyagi, 980-8578, Japan
}

Accepted XXX. Received YYY; in original form ZZZ

\begin{abstract}
Disc galaxies show a large morphological diversity with varying contribution of three major structural components; thin discs, thick discs, and central bulges. Dominance of bulges increases with the galaxy mass (Hubble sequence) whereas thick discs are more prominent in lower mass galaxies. Because galaxies grow with the accretion of matter, this observed variety should reflect diversity in accretion history. On the basis of the prediction by the cold-flow theory for galactic gas accretion and inspired by the results of previous studies, we put a hypothesis that associates different accretion modes with different components. Namely, thin discs form as the shock-heated hot gas in high-mass halos gradually accretes to the central part, thick discs grow by the direct accretion of cold gas from cosmic webs when the halo mass is low, and finally bulges form by the inflow of cold gas through the shock-heated gas in high-redshift massive halos. We show that this simple hypothesis reproduces the mean observed variation of galaxy morphology with the galaxy mass. This scenario also predicts that thick discs are older and poorer in metals than thin discs, in agreement with the currently available observational data.
\end{abstract}

Key words: galaxies:structure - galaxies: formation - galaxies:high-redshift

\section{INTRODUCTION}

Complex structures of disc galaxies pose a tough challenge for galaxy formation theory. Existence of distinct disc and bulge components and their relative dominance constitutes the basis for the traditional Hubble classification of galaxy morphology. Discs are further divided into thin and thick discs with different scale heights (e.g. Burstein 1979; Tsikoudi 1980). Recent studies show that the low-mass galaxies generally contain significant thick discs but their contribution decreases for higher mass galaxies (e.g. Comeron et al. 2014). Various properties such as colours, ages, and metallicities, as well as kinematical properties, are systematically correlated with these respective structural components (e.g. Mo, van den Bosch \& White 2010). Despite a large accumulation of past observational and theoretical studies (e.g. Bruce et al. 2014; Huertas-Company et al. 2015; Brooks et al. 2009), there is not yet a consensus on how various components of disc galaxies were formed throughout the history of the Universe, and when and how the observed diversity of disc galaxy morphology comes out.

Galaxy formation process is greatly controlled by how the gas flows into the dark matter halos and accretes finally onto their central part where stars form. The long-

^ E-mail: noguchi@astr.tohoku.ac.jp (MN) standing paradigm, the shock-heating theory (e.g. Rees \& Ostriker 1977), argues that the gas that entered the halo is heated by a shock wave to a high (virial) temperature, and then gradually accretes to the central part as it cools by emitting radiation (cooling flow). This picture predicts a smooth and continuous gas accretion history. Therefore it is not straightforward to understand why and how the different components of disc galaxies formed, and external origins are invoked in some cases. For example, bulges are sometimes regarded as smaller galaxies swallowed in galaxy mergers (e.g. Zavala et al. 2012).

The shock-heating theory was later modified by the cold accretion scenario which claims that the shock waves do not arise in some cases and a significant part of the gas stays cold, reaching the galaxy in narrow streams almost in a freefall fashion (cold accretion) (e.g. Fardal et al. 2001; Keres et al. 2005; Dekel \& Birnboim 2006). This idea was used to interpret a high incidence of actively star-forming galaxies observed at high redshifts (Dekel et al. 2009). It is also invoked to quench star formation in massive galaxies in cooperation with the feedback from active galactic nuclei in theoretical models (Croton et al. 2006). The predicted cold gas streams are claimed to be found in the form of quasar absorption line systems such as Lyman limit systems (Fumagalli et al. 2011) or Lyman $\alpha$ emitting filaments around forming galaxies (Martin et al. 2016). 
Using a simple model for the gas accretion into a dark matter halo and subsequent cooling and star formation processes, Noguchi (2018b) applied the cold accretion scenario to the Milky Way (MW), and showed that the existence of two chemically different stellar groups observed in the MW disc (e.g. Haywood et al. 2013; Hayden et al. 2015) is naturally explained. In this picture, the stellar group rich (relatively to $\mathrm{Fe}$ ) in $\alpha$ elements $(\mathrm{O}, \mathrm{Mg}, \mathrm{Si}$, etc) was formed by a fast star formation event fuelled by the cold accretion in early times whereas the $\alpha$-poor stellar group was gradually formed by the cooling flow accretion of the shock-heated gas in later epochs. Interestingly, the $\alpha$-rich stars in the solar neighbourhood tend to have larger velocities perpendicular to the disc plane than $\alpha$-poor stars (e.g. Haywood et al. 2013), meaning that they reach to higher altitudes resulting into a thicker configuration. Although this correspondence is not always clear-cut (Haywood et al. 2013), the result of Noguchi (2018b) hints at the association of the cold accretion and the cooling flow with the formation of the MW thick disc and thin disc, respectively (also see Brooks et al. 2009). In more general perspective, we can envisage that a particular mode of gas accretion is associated with a particular component in disc galaxies.

Here we extend this idea and apply this "accretioncomponent correspondence" to the disc galaxy evolution model with various masses, and examine if the cold accretion theory explains the observed characteristics (mass fractions, age, metallicity) of various structural components across galaxy mass. As described below, we take an idealized approach in which the model galaxy evolves "passively" under the various accretion modes ignoring other potentially important physics (e.g., gas recycling by galactic fountains), because we hope to know to what extent the build-up of various galactic structures is governed by the accretion process.

\section{CONNECTING ACCRETION TO COMPONENTS}

The cold-accretion theory predicts three regimes for the thermal state and accretion process of the halo gas depending on the virial mass $\left(M_{\text {vir }}\right)$ of the halo and redshift $z$ as depicted in Fig.1 (Dekel \& Birnboim 2006; Ocvirk, Pichon \& Teyssier 2008). There are two importrant mass scales in defining those regimes. First, $M_{\text {shock }}$ is the virial mass above which a stable shock develops and the halo gas is heated to the virial temperature. Second, $M_{\text {stream }}$ is the mass above which the narrow streams of cold unshocked gas dissapear at high redshifts. Therefore, the cold accretion dominates when $M_{\text {vir }}<M_{\text {shock }}$ (Domain F), whereas the cooling flow dominates in Domain G $\left(M_{\text {vir }}>M_{\text {shock }}\right.$ and $\left.M_{\text {vir }}>M_{\text {stream }}\right)$. The halo gas in Domain H $\left(M_{\text {shock }}<M_{\text {vir }}<M_{\text {stream }}\right)$ has a unique hybrid structure with narrow streams of the cold gas penetrating ambient shock-heated gas. We assume that the cold gas and the shocked gas experience the cold accretion and the cooling flow, respectively. Here we take $M_{\text {shock }}=1.5 \times 10^{11} \mathrm{M}_{\odot}$ and $\log M_{\text {stream }}=9.2+1.067 z$ ( the Supplementary Material for details).

A variant of the evolution model in Noguchi (2018a) was used in order to examine the growth of various components as the halo moves on this plane (see the Supplementary Material for details). In short, the original model was

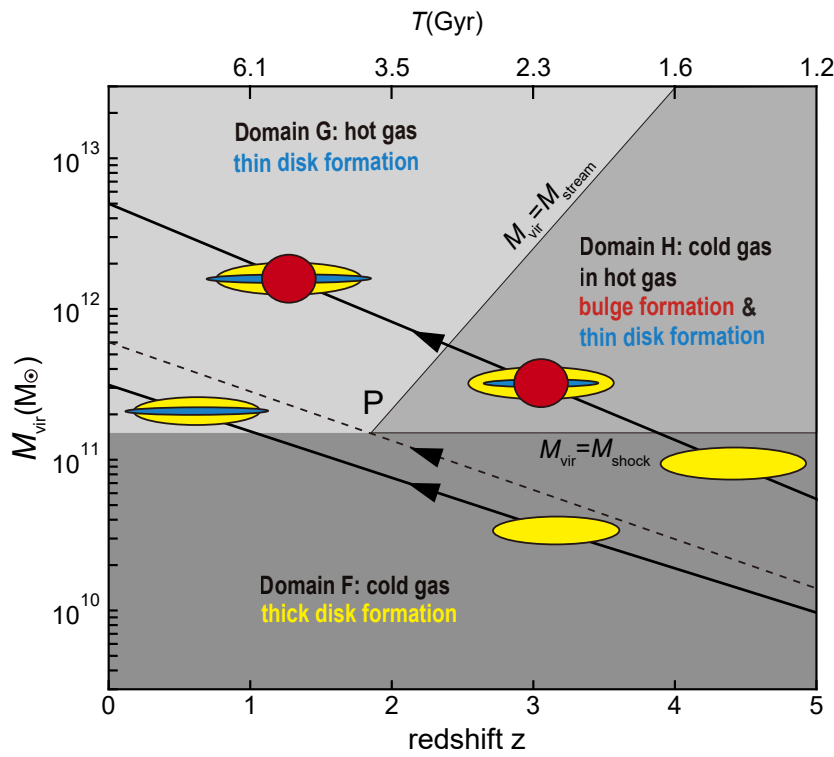

Figure 1. Three different states of the halo gas according to the cold-accretion theory. $M_{\mathrm{vir}}, z$, and $T$ denote the virial mass of the galaxy, redshift, and cosmic time, respectively. $\boldsymbol{M}_{\text {shock }}$ and $\boldsymbol{M}_{\text {stream }}$ are the mass scales explained in the text. Formation of thin disc (blue), thick disc (yellow), and bulge (red) is indicated schematically. Two solid diagonal lines indicate the evolutionary path of the least and most massive galaxies studied here. The present virial masses for these galaxies are $M_{\mathrm{vir}, 0}=3.05 \times 10^{11} \mathrm{M}_{\odot}$ and $4.98 \times 10^{12} \mathrm{M}_{\odot}$, respectively. The dashed line indicates the galaxy which passes through the point $\mathrm{P}$, i.e., the intersection of the $M_{\text {shock }}$ line and $M_{\text {stream }}$ line. The present virial mass of this galaxy (which we call "the critical mass") is $M_{\text {critical }}=5.72 \times$ $10^{11} \mathrm{M}_{\odot}$.

simplified by ignoring the internal structure of the galaxy ("one-zone model") with each galactic component at every moment specified only by its mass. The gas accretion rate to the central galaxy is calculated as a function of time and we apply "accretion-component correspondence" in order to trace the mass growth of each galactic component. As discussed above, we assume that the gas accreted in unheated state (Domain F) ends-up in the thick disc, whereas the gas supplied by the cooling flow (Domain G) is used to grow the thin disc. The fate of the gas in Domain $\mathrm{H}$ is more elusive. By the reason discussed below, we associate this domain with the bulge formation. Galactic bulges, including that of MW, are complex structures (e.g. Ness et al. 2013), and their ori$\operatorname{gin}(\mathrm{s})$ is not yet clear (e.g. Rojas-Arriagada et al. 2017). One possibility is that the gravitationally unstable gas-rich discs in young galaxies develop star-forming dense clumps, which subsequently sink toward the galactic center due to dynamical friction to form a bulge as first proposed by Noguchi (1998). Alternatively, clumps may form in the cold gas filaments feeding the galaxy (Dubois et al. 2012). This kind of scenarios rely on the longevity of the clumps, which are constantly subject to disruption by feedbacks from star formation inside. The hybrid nature of the halo gas in Domain $\mathrm{H}$ may help those clumps survive longer owing to high external pressure exerted by the surrounding hot gas component. Indeed, cosmological simulations for disc galaxy formation 
suggest the confinement of cold gas stream by large pressure of the shock-heated gas is a generic phenomenon. In Figs.4 and 5 of Keres et al. (2009) and Fig.10 of Ness et al. (2013), the streams of cold gas are seen to get thinner once they enter the region occupied by the hot shock-heated gas while they reach to the galactic center mostly maitaining their widths in the absence of the hot gas. Also the overdensity of the cold gas is much larger than that of the hot gas in massive galaxies (e.g. van de Voort \& Schaye 2012), suggesting that the cold streams are squeezed by the high pressure of the shock-heated gas. Clumpy structures are ubiquitous in cold streams. In isolation, they will disrupt once a certain amount of stars form in them. However, in the high-pressure environments, they will survive to reach the central parts of the halos. Based on this consideration, we assume that the cold gas in Domain H ends up in bulges eventually whereas the cooling flow of the shock-heated gas in Domain $\mathrm{H}$ contributes to thin disc formation.

By varying the present host halo virial mass $M_{\mathrm{vir}, 0}$, we examined how the growth of each structural component depends on the galaxy mass. Nine models are calculated with $\log M_{\text {vir,0 }}$ equally spaced between the lowest and highest values indicated in Fig.1. Time step of $7 \times 10^{6}$ years was used to evolve the model, starting at $z=37$. The present total stellar mass of the least and most massive galaxies are $M_{\text {star }}=2.13 \times 10^{9} \mathrm{M}_{\odot}$ and $1.50 \times 10^{11} \mathrm{M}_{\odot}$, respectively. The "critical" model is defined as the model which passes through the intersection of the $M_{\text {shock }}$ line and $M_{\text {stream }}$ line (the dashed line in Fig.1), and its present stellar mass is $M_{\text {star }}=6.91 \times 10^{9} \mathrm{M}_{\odot}$.

\section{RESULTS}

Star formation history of the most and least massive galaxies in the model is shown in Fig.2. As shown in Fig.2a, low-mass galaxies with $M_{\mathrm{vir}, 0}<M_{\text {critical }}$ first grow thick discs by the cold-mode accretion. After crossing the shock-heating border (i.e., $M_{\text {vir }}=M_{\text {shock }}$ ), the gas accretion is switched to the cooling flow (Fig.1), and thin discs are formed. Fig.2c shows that the mass fraction of the thick disc decreases monotonically with time. The massive galaxies with $M_{\mathrm{vir}, 0}>M_{\text {critical }}$ evolve in a more complicated way (Fig.2b). After the thick disc formation in Domain F, they enter the hybrid Domain $\mathrm{H}$ and develop bulges (Fig.1). Finally thin discs start to dominate when they leave this domain. Galactic bulges in this scenario are not the first mass component to emerge but thick discs predate bulges. Therefore, the bulge mass fraction is initially almost zero and then starts to increase as the bulge develops. In later phase $(\mathrm{T}>7 \mathrm{Gyr})$, it decreases as the thin disc grows (Fig.2d).

Fig.3 summarizes the morphological observation performed by Comeron et al. (2014) which is the most extensive one at present. Of the sample galaxies plotted in Fig.3, those with bulges are dominated by classical bulges and/or unresolved central mass components. We combined these components into "bulge" for comparison with the model. A small number of galaxies with pseudo-bulges were excluded but including them does not make any significant changes. Boxy or peanut-shaped bulges were excluded because they are considered to be bar structures seen edge-on and therefore of disc origin.
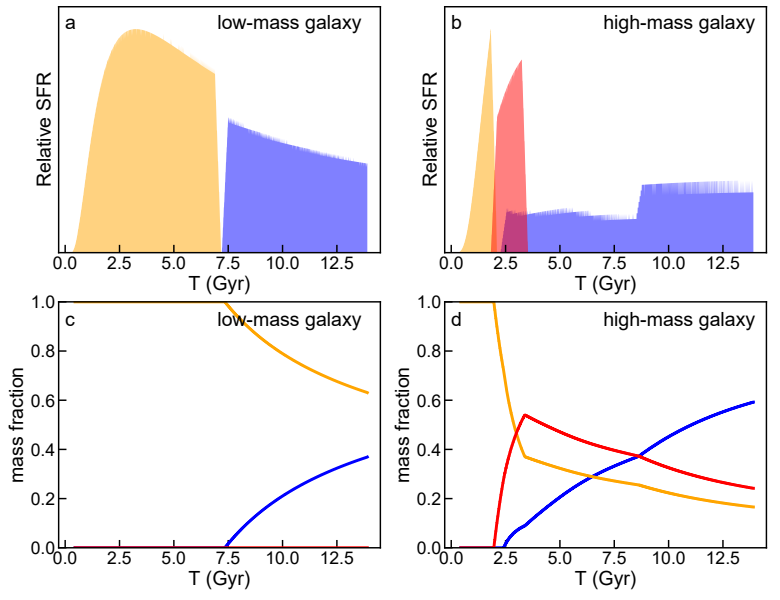

Figure 2. Panels a,b: Star formation rate (SFR) in the least massive and the most massive galaxies. Blue, orange, and red indicate SFR for thin disc, thick disc, and bulge, respectively. SFR is averaged over 20 successive time steps $(0.28 \mathrm{Gyr})$ to smooth out the short timescale fluctuations arising in the model calculation, and given in arbitrary units. Panels c,d: Time variation of the mass fraction relative to the total stellar mass of thin disc (blue), thick disc (orange), and bulge (red).

Fig.3a demonstrates that the model reproduces the absolute masses of various components as a function of the total galaxy stellar mass for the present-day disc galaxies reasonably well, although the observation shows a large scatter. Note that the model describes the mean behavior and does not take into account possible dispersions in the dark matter halo properties at the same virial mass. Both in the model and observations, the bulge mass shows the steepest increase as the total galaxy mass increases, while the thick disc mass increases mildly. The increase of the thin disc is the intermediate one. Figs.3b,c, and d plot mass fractions and ratios against the galaxy mass. The model agrees with the observation also regarding relative dominance of the different components. Low-mass galaxies $\left(M_{\text {star }}<10^{10} \mathrm{M}_{\odot}\right)$ lack bulges or have only small bulges, with thick discs occupying comparable masses with thin discs (Fig.3b). Galaxies with larger masses contain increasingly more massive bulge components but the contribution of thick discs decreases. The mass ratio of thick and thin discs decreases monotonically with the total stellar mass (Fig.3c), while the bulge-to-total mass ratio increases (Fig.3d). These results are consistent with the behavior of star formation rate in Fig.2 showing that massive galaxies experience more intense cooling flow accretion in Domain G contributing to the thin disc growth in $T>8.5$ Gyr. They also experience an additional hybrid accretion in Domain $\mathrm{H}$, which leads to the bulge formation by the cold gas flow around $T=3 \mathrm{Gyr}$ and the thin disc growth by the cooling flow of the shocked gas during the period 2.5 Gyr $<T<8.5$ Gyr.

The two kinds of discs are different also in age and chemical compositions as shown in Fig.4. Fig.4a shows that the model thick discs are old components with most stars formed more than 10 Gyr ago, whereas thin discs consist of much 

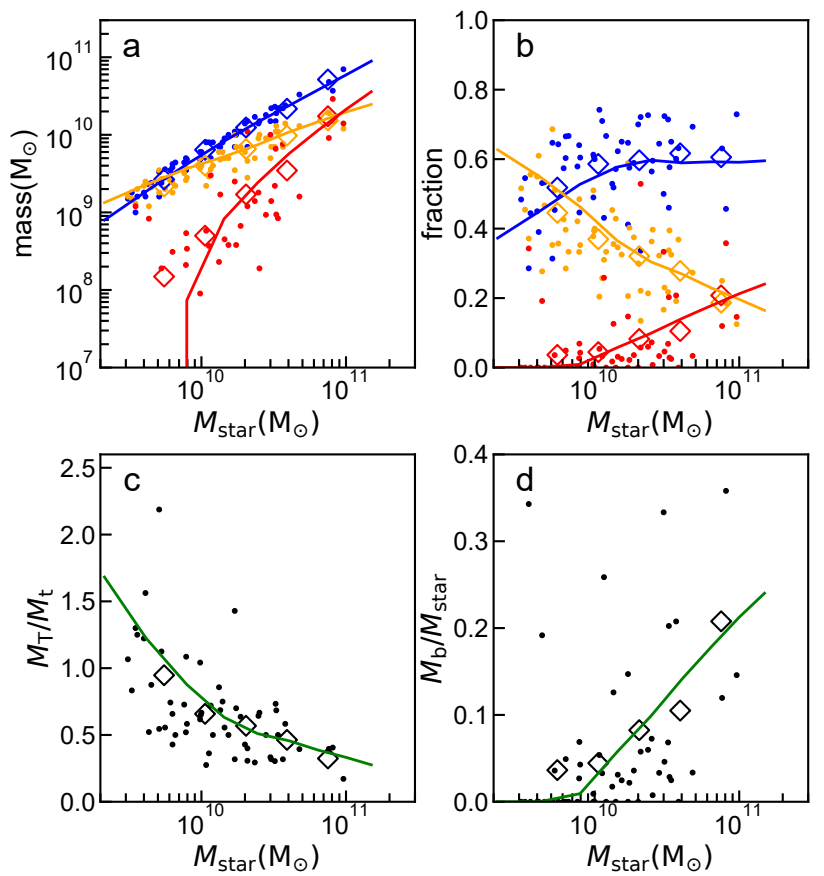

Figure 3. Masses of various structural components and their ratios plotted against the total stellar mass of the galaxy. The total stellar mass, $M_{\text {star }}$, is the sum of the masses of thin disc $\left(M_{\mathrm{t}}\right)$, thick disc $\left(\boldsymbol{M}_{\mathrm{T}}\right)$, and bulge $\left(\boldsymbol{M}_{\mathrm{b}}\right)$. Solid lines show model results. The lowest and highest stellar masses in the model are $M_{\text {star }}=2.13 \times 10^{9} \mathrm{M}_{\odot}$ and $1.50 \times 10^{11} \mathrm{M}_{\odot}$, respectively. Observed values by Comeron et al. (2014) are indicated by dots, with diamonds showing the mean in each mass-bin. Panel a: Mass of each component (thin disc: blue, thick disc: orange, bulge: red). Panel $\mathrm{b}$ : The same as Panel a, but for the mass fraction of each component. Panel c,d: Mass ratios involving the indicated components.

younger stars, in broad agreement with the observations for the solar neighbourhood and for other galaxies. The chronological order of thick and thin disc formation is also reflected in their chemical properties. The model thin discs include more iron relative to hydrogen than the thick discs (Fig.4b) because they are formed from the interstellar gas polluted by iron produced and released by the supernovae originating in early star formation which made the thick discs. Solar neighbourhood shows a similar trend, though the data for other galaxies show large scatters and no clear trend.

We proposed here that the galactic thick discs were formed by the cold accretion. Other proposed mechanisms include the stirring-up of the existing thin discs by mergers with smaller galaxies (e.g. Quinn, Hernquist \& Fullagar 1993) and the radial migration of kinematically hot stars from inner galactic regions to the solar neighbourhood (Minchev, Chiappini \& Martig 2013). It seems, however, difficult to explain the distinct chemical bimodality of MW disc stars in these scenarios. Although several processes may contribute to the thick disc formation actually, the successful reproduction of structural variations (Fig.3) makes the coldflow origin of thick discs a viable hypothesis, in combina-
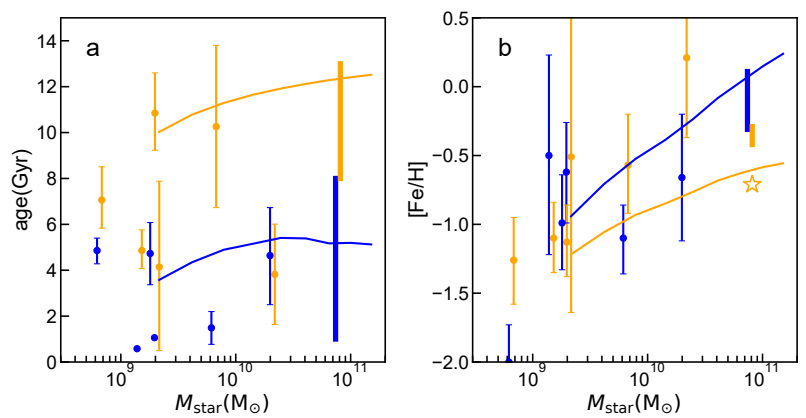

Figure 4. Age and iron abundance of the thick (orange) and thin (blue) discs plotted against the total stellar mass of the galaxy. Solid curves indicate the model. Dots with error bars indicate the observations for external galaxies (thick disc values are slightly displaced horizontally for clarity) by Yoachim \& Dalcanton (2008), with the galaxy circular velocity converted to the stellar mass using the Tully-Fisher relation by Reyes et al. (2011). Panel a: The mean stellar age. The vertical thick segments indicate the age range estimated for the solar neighbourhood data by Haywood et al. (2013). Panel b: The iron abundance ratio. The vertical thick segments indicate the range for the solar neighbourhood data by Hayden et al. (2015). The star symbol indicates the abundance of the solar neighborhood thick disc observed by Reddy, Lambert \& Prieto (2006).

tion with its ability to explain the age and chemical duality demonstrated in Noguchi (2018b). Brooks et al. (2009) investigated the accretion history of the different components of the numerically simulated disc galaxies and showed that the shock heated gas tends to end-up in thin discs, while the thick discs and bulges are predominantly formed from the gas accreted in unheated states. Bulges also contain significant contributions from clump accretion. The present model is thus in broad agreement with the cosmological simulation by Brooks et al. (2009), although the latter does not discuss the change of accretion mode in relation to the growth of the host dark matter halo.

\section{IMPLICATION FOR HIGH-REDSHIFT GALAXIES}

Present study predicts a large structural variety of highredshift galaxies. The particularly interesting redshift range is $2<\mathrm{z}<3$, for which we will witness three kinds of evolutionary status (Fig.1). Galaxies with the smallest masses will be in Domain F and making thick discs. Intermediatemass galaxies, staying in Domain $\mathrm{H}$, will be forming bulges in addition to the already-formed thick discs. Finally, the most massive galaxies will have already entered Domain G, creating thin discs. Present-day observations can reach such high redshifts, providing interesting new insights. For example, Tacchella et al. (2015) examined the star formation activity in galaxies at $z \sim 2$ and found that more massive galaxies show stronger quenching (i.e., cessation of star formation) in their central parts, hinting at the existence of matured bulges. This seems to agree partly with the above prediction. For more quantitative comparison, Fig.5 shows how the model galaxies build-up bulge components as they 


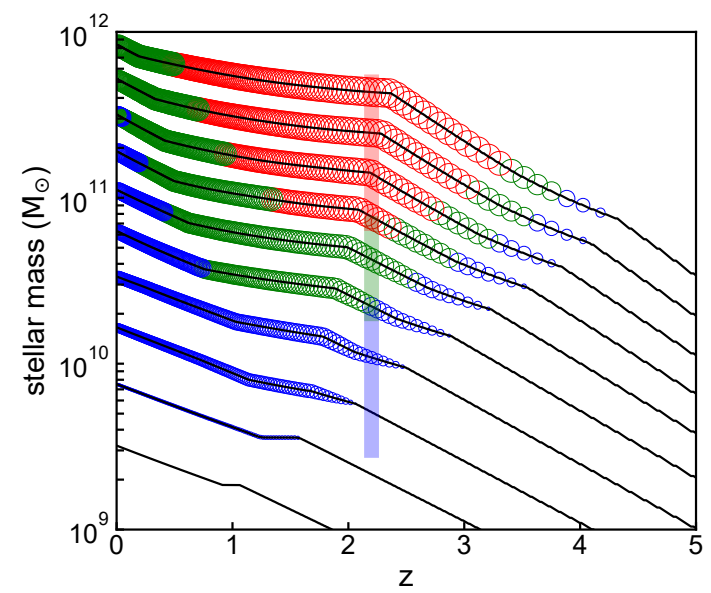

Figure 5. Evolution of the bulge-to-total stellar mass ratio for each model. Black lines show the evolution of the total stellar mass. Size of the circle is proportional to the bulge mass ratio, $f_{\mathrm{b}} \equiv M_{\mathrm{b}} / M_{\text {star }}$, which is also colour-coded (blue: $f_{\mathrm{b}}<0.3$, green: $0.3<f_{\mathrm{b}}<0.5$, red: $\left.0.5<f_{\mathrm{b}}<0.8\right)$. For this figure, the mass range of the halo is $3.8 \times 10^{11} \mathrm{M}_{\odot}<M_{\text {vir }, 0}<2.5 \times 10^{13} \mathrm{M}_{\odot}$, equaly spaced in $\log \boldsymbol{M}_{\mathrm{vir}, 0}$. Shaded segments indicate the observations by Tacchella et al. (2015) for $\mathrm{z}=2.2$ galaxies with the mean Sersic indices $n$ colour-coded (blue: $n=1$, green: $n=1.9$, red: $n=2.8$ ).

grow in the stellar mass. Tacchella et al. (2015) measured the Sersic indices $n$ of the stellar density profile instead of the bulge mass fraction, $f_{\mathrm{b}} \equiv M_{\mathrm{b}} / M_{\text {star }}$. Based on the result by Vika et al. (2014), we make a crude estimate that $n=1,1.9$ and 2.8 correspond to $f_{\mathrm{b}}=0.2,0.4$ and 0.6 , respectively. Once this conversion is made, the observation by Tacchella et al. (2015) agrees reasonably well with the model, with both showing the increasing dominance of the bulge for more massive galaxies. In the present model, the bulge mass ratio attains a peak at $z \approx 2$, decreasing toward smaller redshifts due to the growth of the thin disc and toward higher redshifts because thick discs predate the bulges. Detection of this change both along the evolutionary tracks and at the fixed stellar masses by future observations will further strengthen the scenario proposed in the present study.

\section{CONCLUSIONS}

We seek the origin of different structural components of disc galaxies in the different physical states of their raw material gas when it is distributed in the host halo. We associate the shock-heated hot gas in massive halos with the thin disc components and the cold unshocked gas in low mass halos with the thick discs. Further, we propose a new formation channel of the bulges from the cold gas compressed by the surrounding shock-heated hot gas in massive galaxies at high redshifts. Calculation of the build-up process of each component for a wide galaxy mass range based on this proposed correspondence reproduces the increasing dominance of the bulges and thick discs toward higher and lower galaxy masses, respectively. This scenario also explains the age and metallicity difference of thin and thick discs observed in the Milky Way and other disc galaxies.

\section{ACKNOWLEDGEMENTS}

We acknowledge A. Faisst for providing data for the mass fraction of gas for star forming galaxies. We thank anomymous referees for useful comments which helped us improve the paper.

\section{REFERENCES}

Bruce V. A. et al., 2014, MNRAS, 444, 1001

Brooks A. M. et al., 2009, ApJ, 694, 396

Burstein D., 1979, ApJ, 234, 829

Comeron S. et al., 2014, A\&A, 571, 58

Croton D. J. et al., 2006, MNRAS, 365, 11

Dekel A., Birnboim Y., 2006, MNRAS, 368, 2

Dekel A. et al., 2009, Nature, 457, 451

Dubois Y. et al., 2012, MNRAS, 423, 3616

Fardal M. A., 2001, ApJ, 562, 605

Fumagalli M. et al., 2011, MNRAS, 418, 1796

Hayden M. R., 2015, ApJ, 808, 132

Haywood M. et al., 2013, A\&A, 560, 109

Huertas-Company M. et al., 2015, ApJ, 809, 95

Keres D. et al., 2005, MNRAS, 363, 2

Keres D. et al., 2009, MNRAS, 395, 160

Martin D. C. et al., 2016, ApJ, 824, L5

Minchev I., Chiappini C., Martig M., 2013, A\&A, 558, 9

Mo H., van den Bosch F., White S., Galaxy Formation and Evolution (Cambridge Univ. Press, New York, 2010)

Nelson D. et al., 2013, MNRAS, 429, 3353

Ness M. et al., 2013, MNRAS, 432, 2092

Noguchi M., 1998, Nat, 392, 253

Noguchi M., 2018a, ApJ, 853, 67

Noguchi M., 2018b, Nat, 559, 585

Ocvirk P., Pichon C., Teyssier R., 2008, MNRAS, 390, 1326

Quinn P. J., Hernquist L., Fullagar D. P., 1993, ApJ, 403, 74

Reddy B.E., Lambert D.L., Prieto, C.A., 2006, MNRAS, 367, 1329

Rees M. J., Ostriker J. P., 1977, MNRAS, 179, 541

Reyes R. et al., 2011, MNRAS, 417, 2347

Rojas-Arriagada A. et al., 2017, A\&A, 601, 140

Tacchella S. et al., 2015, Science, 348, 314

Tsikoudi V., 1980, ApJS, 43, 365

van de Voort F., Schaye J., 2012, MNRAS, 423, 2991

Vika, M. etal., 2014, MNRAS, 444, 3603

Yoachim P., Dalcanton J. J., 2008, ApJ, 683, 707

Zavala J. et al., 2012, MNRAS, 427, 1503

This paper has been typeset from a $\mathrm{T}_{\mathrm{E} X} / \mathrm{LAT}_{\mathrm{E}} \mathrm{X}$ file prepared by the author. 